\title{
The role of SIPA1 in the development of cancer and metastases (Review)
}

\author{
CHANG LIU, WEN GUO JIANG, RACHEL HARGEST and TRACEY AMANDA MARTIN \\ Cardiff China Medical Research Collaborative, School of Medicine, Cardiff University, Cardiff CF14 4XN, United Kingdom
}

Received November 12, 2019; Accepted June 16, 2020

DOI: $10.3892 / \mathrm{mco} .2020 .2102$

\begin{abstract}
Cancer is a leading cause of mortality and the majority of deaths are due to metastases. Many molecules have been implicated in the development of metastases. Signal induced proliferation associated protein 1 (SIPA1), a mitogen-inducible gene, has been demonstrated to be involved in the metastasis of various solid tumours and may indicate a poor prognosis. Polymorphisms of SIPA1 can be associated with several different types of cancer and interactions between SIPA1 and binding molecules integrate a series of cellular functions, which may promote the development and metastasis of cancer. The mechanisms by which SIPA1 promotes the development and metastasis of cancer varies among tumour types. The present review describes the structure, function and regulation of SIPA1 and focuses on its role in cancer metastasis. Possibilities for future research and the clinical application of SIPA1 are also discussed.
\end{abstract}

\section{Contents}

1. Introduction

2. Cloning and identification of the SIPA1 gene

3. The structure and expression of SIPA1 protein

4. Germline polymorphisms in SIPA1

5. SIPA1 family and Rap1-GTPase activating proteins

6. SIPA1 interacting molecules

7. The multiple roles of SIPA1 in different cancer types

8. Conclusion

\section{Introduction}

Since Dr. Stephen Paget proposed the 'seed and soil' theory of metastasis in 1889 (1), the mechanism of cancer metastasis has been an area of interest (2). Cancer metastasis has been shown to be a complex process involving multiple factors including sustaining proliferative signalling and evading growth suppressors, resisting cell death, enabling replicative immortality, inducing angiogenesis, activating invasion, migration and drug resistance $(3,4)$. Increasing numbers of oncogenes, such as ErbB2, PI3KCA, MYC, CCND1 and tumour suppressor genes, such as p53 and $\mathrm{Rb}$ have been identified as being related to tumour metastasis (5). Additionally, many more signalling pathways have been found to play an essential role in the development and progress of cancer including TGF- $\beta$ (6), MAPK (7), Wnt (8), NOTCH (9), Fak (10), PI3K/Akt $(11,12)$. Among all these oncogenes, signal-induced proliferation-associated protein 1 (SIPA1), a mitogen-inducible gene encoding a GTPase-activating protein for Rap1 and Rap2, was identified as an important moderator participating in several cancer related signalling pathways (13). In this paper, we focus on SIPA1 the structures of SIPA1 gene and protein, the relationships between polymorphisms of SIPA1 and tumour susceptibility, the interaction between SIPA1 and other molecules and the functions of SIPA1 in solid tumours.

\section{Cloning and identification of the SIPA1 gene}

The SIPA1 gene, also known as SPA1 (suppressor of phyA-105), was first cloned in 1995 from a murine lymphoid cell line, LFD 14, after IL-2 stimulation (14). SIPA1 in mice was originally described as 3,518 bp long with a long open reading frame (ORF) (from position 1,199 to 3,280) and several short ORFs at the 5'-end (14). In 1997, the same research team cloned the human SIPA1 cDNA from human peripheral blood lymphocytes (PBL) after stimulation with phytohemagglutinin and TPA (15). The human SIPA1 gene was mapped to chromosome 11q13.1, spanning $12.8 \mathrm{~kb}$. Human SIPA1 gene is highly homologous to the murine gene, containing 16 exons, amongst which exon 1,91 bp of exon 2 (considered as 5'-UTR) and the 3'-region 205 bp of exon 16 are untranslated (13). The human SIPA1 genome contains a much longer ORF of 1,042 residues (from position 297 to 3,425 ) (15). In the 5' terminal region of SIPA1 gene, residues from position 192 to 539 (containing part of the exon 5, exon 6,7 and part of exon 8 in human) were found

Key words: signal induced proliferation associated protein 1 , cancer, tight Junctions, metastasis 
to be highly homologous to human Rap1-GAP (Gap related domain, GRD). Upstream of GRD there is a proline-rich domain with the potential ability to bind $\mathrm{SH} 3$ and downstream of GRD there is the PDZ domain which consists of part of exon 9. The 3 ' region, part of exon 14, exon 15 and translated part of exon 16 encode a leucine zipper (LZ)-like domain, which was also found to be conserved in both humans and mice $(13,15)$.

\section{The structure and expression of SIPA1 protein}

In mice, the SIPA1 gene encodes a $68 \mathrm{KDa}$ protein (P68) with 693 amino acids, mostly located in the nuclei (14), whilst the human SIPA1 protein contains 1,042 amino acids with a molecular mass of $130 \mathrm{KDa}$ (15). Three domains were identified in the SIPA1 protein: i) RapGTPase-activating protein (GAP) related domain (GRD) (350-539); ii) PDZ domain (685-759); iii) Leucine zipper like (LZ) domain (964-1042) which is similar to myosin tail (13).

There is a proline-rich domain including possible SH3-binding motifs located in the $\mathrm{N}$ terminal to the GRD and upstream the LZ domain has a probable PEST sequence (Fig. 1) (15). Expression levels and localization of SIPA1 protein vary in different human tissues and cells. SIPA1 protein is most highly expressed in the lymphohematopoietic system such as spleen, bone marrow and thymus. SIPA1 may be located in the cytoskeleton, plasma membranes and nuclei depending on the type of cell and SIPA1's interaction with other proteins (13).

\section{Germline polymorphisms in SIPA1}

There are thousands of single-nucleotide polymorphisms (SNPs) in the SIPA1 gene, of which seven SNPs located in the promoter or encoding regions of SIPA1 have been identified as being associated with tumourigenesis, metastasis and prognosis in previous studies (16-20). Amongst them, most research has focused on three SNPs: i) rs931127, a G>A SNP located in the promoter region; ii) rs 3741378 , a $\mathrm{C}>\mathrm{T}$ or $\mathrm{C}>\mathrm{G}$ SNP which encodes for the replacement of a serine (Ser) to phenylalanine (Phe) amino acid in exon 3; iii) rs746429, a G>A SNP which encodes for a synonymous amino acid (Alanine, Ala) transformation in exon14.

These three SNPs are considered to be related to the development, metastasis and prognosis of breast, lung and cervical cancer (16-25).

Other SNPs have been shown to be involved in breast cancer: rs2306364, a G>A SNP rs2448490, a G $>A, G>C$ or $\mathrm{G}>\mathrm{T}$ SNP, both of which encode for a synonymous amino acid (Alanine, Ala) transformation $(16,19,25)$ In addition, rs75894763, a G>A SNP also encoding for a synonymous amino acid (Valine, Val) transformation (16) and rs75894763 have been shown to be related to lung cancer (25). The G>T SNP, rs3741379, encoding for the replacement of an Ala to Ser amino acid has been reported to be involved in the process of lung cancer metastasis (25).

\section{SIPA1 family and Rap1-GTPase activating proteins}

The initial function of SIPA1 was believed to be specific GAP activity for Ras-related mediating proteins, Rap1, Rap2,
Rsr1 and nuclear Ran (15), although recent research suggests that SIPA1 cannot work as a GAP for Ran or other small GTPases (15). SIPA1 overexpression induces rounding and eventual detachment of inherently adherent cells from the extracellular matrix by inhibiting endogenous Rap1 activation, indicating that Rap1 signals are involved in the regulation of cell adhesion and SIPA1 functions as a negative regulator of cell adhesion (14).

Human RapGTPase activating protein mainly consists of two subfamilies: i) SIPA1 family; ii) rapGAP family.

In addition to SIPA1, the SIPA1 family also includes SIPA1-like1 (SIPAL1, also called E6TP1 or SPAR), SIPAL2, and SIPAL3; and the rapGAP family contains rapGAP1 and rapGAP2 (13). All the RapGAPs share a homologous catalytic GRD domain in addition to an analogous PDZ domain $(13,15)$.

\section{SIPA1 interacting molecules}

Nuclear SIPA1 and integrin $\beta 1$. Nuclear SIPA1 has been shown to interact with a number of other proteins (Fig. 2) and activate the integrin $\beta 1$ promoter in breast cancer cells (26). Nuclear SIPA1 has been shown to interact with and activate the integrin $\beta 1$ promoter in breast cancer cells (26). After SIPA1 knock down in MDA-MB-231 cells, mRNA levels of SIPA1 were significantly downregulated. Chromatin immunoprecipitation experiments revealed that nuclear SIPA1 could interact with the ITGB1 promoter and increase its transcription activity, causing phosphorylation changes in the integrin-mediated FAK/Akt signalling pathway, thereby affecting the adhesion and invasion capability of cancer cells (26).

Association of SIPA1 and AF6. Co-immunoprecipitation experiments demonstrated that SIPA1 bonded specifically with AF6 (afadin) in 293T cells transfected with both SIPA1 and AF6. Further studies revealed that the binding occurred between the GRD domain of SIPA1 and the PDZ domain of AF6. AF6 was reported to localize at cell adhesion sites and have an association with the tight junction (TJ) protein ZO-1 (Zonula Occludens 1), SIPA1 also co-localized with AF6 in similar cell to cell adhesion sites, so it was suggested that SIPA1 may also regulate TJs via AF6, although there is only scant evidence for this (27).

SIPA1 colocalizes with AQP2 in renal collecting ducts. Studies have shown that in renal collecting ducts, SIPA1 directly binds to aquaporin-2 (AQP2) and is involved in the regulation of trafficking $\mathrm{AQP} 2$ to the apical membrane. AQP2 has the ability to bind to molecules containing a PDZ domain, so SIPA1 with a PDZ domain can combine with AQP2 and play a role in the intracellular transport of AQP2. Studies also demonstrated that Rap1 signalling pathway was involved in the AQP2 intracellular transport system. As a regulation factor of Rap1, SIPA1 can control the transport process by inhibiting Rap1. Therefore the interaction between SIPA1 and AQP2 may be both direct and indirect (28).

Brd4 interacts with SIPA1. In HeLa cells, the bromodomain protein Brd4 was found to bind to the GRD domain of SIPA1 directly and this interaction is mainly located in the cell 


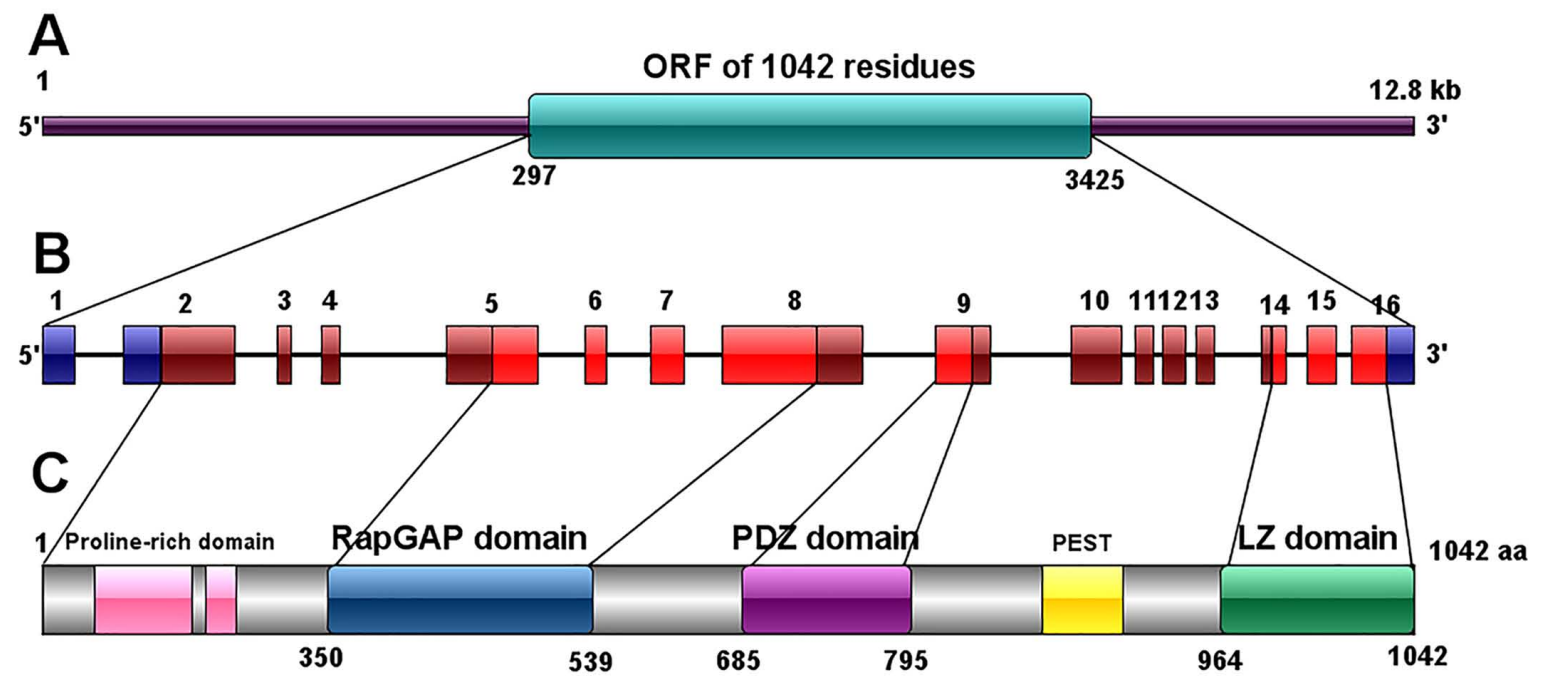

Figure 1. Structure of the human SIPA1 gene and protein. (A) Schematic representation of the SIPA1 gene structure. (B) The 16 exons of the SIPA1 gene. (C) Schematic representation of the SIPA1 protein structure. SIPA1, signal induced proliferation associated protein 1.

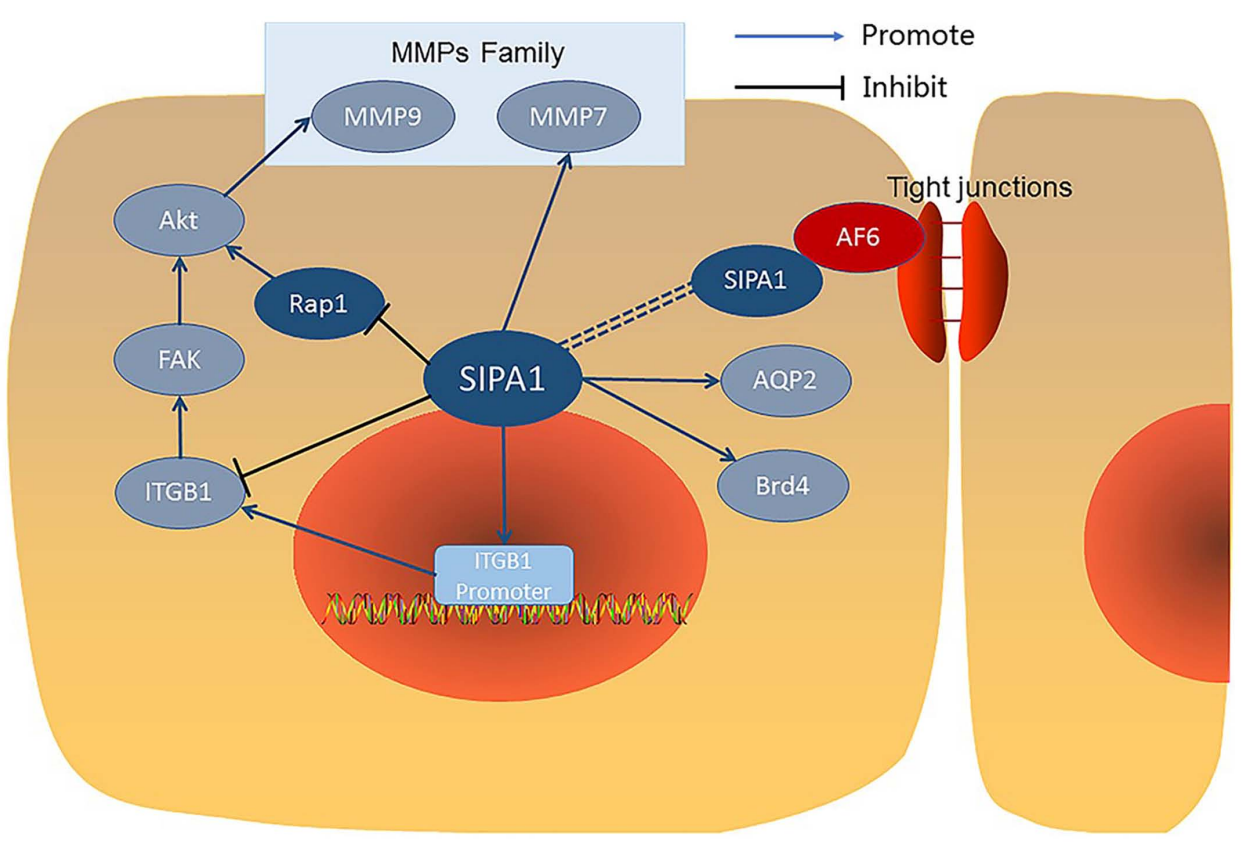

Figure 2. Schematic diagram presenting the interacting molecules of SIPA1. SIPA1 is central to the presented interactions. SIPA1 regulates the transcription of ITGB1 and controls proteins of the MMP family. Rap1 inhibition may modulate MMP via Akt signalling. AF6, presented in red, is the key molecule by which SIPA1 directly affects and adjusts tight junction proteins, maintaining cell-to cell barrier function. SIPA1, signal induced proliferation associated protein 1; ITGB1, integrin $\beta 1$ promoter; MMP, matrix metallopeptidase; AF6, afadin.

nucleus especially at or near the inner aspect of the nuclear membrane and enhanced the RapGTPase activity of SIPA1 for Rap1 and Rap2 (29,30). The combination of SIPA1 and Brd4 promotes the cell cycle through M to G1 phases, which may also regulate cancer development (30).

SIPA1 is a metastasis efficiency modifier Mtes1. Genetic mapping revealed thar SIPA1 a candidate modifier for the Mtes1 (Metastasis efficiency suppressor gene 1), which locates on the mouse chromosome 19 and contains a sequence of human's metastasis suppressor gene Brms1 (31). In mice, SIPA1 works as a candidate for underlying Mtes1. The mice kidney cancer cell line cos-7 cells with the SIPA1 protein which had alanine (A) (SIPA1/741A) at the amino acid position 741 showed higher metastatic potential than those which had threonine (T) (SIPA1/741T) at the Position 741 in the PDZ domain (31). The higher metastatic potential could be induced through the mechanism that the Rap1GAP activating level was higher in the cells with SIPA1/741A SNP than those with SIPA1/741T SNP (31).

\section{The multiple roles of SIPA1 in different cancer types}

Breast cancer. Most research on the role of SIPA1 in cancer and cancer metastasis has focused on breast cancer. Immunohistochemical staining has shown that in breast cancer 
patients, SIPA1 localized to the nuclear region. Its expression level may also be a predictive factor for lymph node metastatic status. Similarly, in breast cancer cell lines, SIPA1 is mainly localized to the nucleus in the aggressive breast cancer cell line MDA-MB-231 cells after transduction to overexpress SIPA1 (26). In vitro cell function experiments demonstrated that knockdown of SIPA1 reduced adhesion, migration and invasion in the MDA-MB-231 breast cancer cell line, but promoted the cells to proliferate (26). These changes may be due to the fact that nuclear SIPA1 interacts with and activates the integrin $\beta 1$ promoter (ITGB1), thereby regulating the adhesion and invasion of breast cancer cells. Knockdown of SIPA1 in the MDA-MB-231 cell line suppressed the integrin mediated FAK/Akt-MMP9 signal pathway by markedly decreasing the phosphorylation levels of FAK and Akt and extracellular secretion of MMP9 (26). Thus the mechanism of SIPA1 promoting breast cancer cell adhesion, invasion and metastasis may occur by regulating the integrin $\beta 1$ /FAK/Akt-MMP9 signalling pathway.

Prostate cancer. In patients with prostate cancer $(\mathrm{CaP})$, high expression of SIPA1 was associated with poor prognosis and tumour metastasis (32). Similarly in the human CaP cell lines, LNCaP with low metastatic capacity inoculated in SCID mice was accompanied by undetectable levels of SIPA1, while PC 3 cells with high metastatic capacity in SCID mice was accompanied by high levels of SIPA1 expression (32). After transduction of SIPA1, the low metastatic LNCaP cells inoculated into the testis of SCID mice exhibited a propensity to metastasize to the abdominal lymph nodes. Following knockdown of SIPA1, the highly metastatic PC 3 cells exhibited reduced metastatic potential and there was no significant change in the size of primary tumour in both groups (32). Thus the effect of SIPA1 on prostate metastasis is greater than the effect on proliferation. In vitro, after transduction of SIPA1 in LNCaP cells, expression of SIPA1 resulted in decreased adhesion of $\mathrm{CaP}$ cells to the extracellular matrix (ECM). Nuclear Brd4 and ECM-related gene expression were down-regulated, which was regulated by Rap1 activation (32). A meta-analysis of human gene expression data (Oncomine website: https://www.oncomine.org/resource/login.html) from prostate, lung and a variety of solid tumours showed overexpression of SIPA1 in human primary prostate cancer tissues which was related to cancer metastasis progress.

Oral squamous cell carcinoma. Expression of SIPA1 in both human oral squamous cell carcinoma (OSCC) and OSCC cells is higher than in normal tissue, and is related to regional lymph node metastasis in OSCC patients. In the OSCC cell line HSC-3 and HSC-4, the knockdown of SIPA1 reduced the ability of cells to invade and migrate, but increased the adhesion of cells and had no significant effect on proliferation compared with the control group. In the same cell line, knockdown of SIPA1 down-regulated the cytosolic expression of BRD4, but abundant BRD4 protein was still expressed in the nucleus (33). The interaction between SIPA1 and BRD4 may promote OSCC metastasis. Expression of ITGB1, an integrin which is known to be an important marker in cell invasion and adhesion, was significantly higher in SIPA1 knock down cells than control cells. However, expression of MMP7 (membrane metalloproteinase 7) which has an essential role in tumour invasion, growth and metastasis, was markedly reduced after knockdown of SIPA1 (33). Therefore, SIPA1 and its interaction with BRD4 may impact the development and metastatic progression of OSCCs by regulating ITGB1 and MMP7.

Colorectal cancer. In human colorectal adenocarcinoma patients, there was increased expression of SIPA1 in tumours, especially in well-differentiated and moderately differentiated tumours compared to poorly differentiated tumours. Patients with a higher expression level of SIPA1 had a poor prognosis. In vitro experiments demonstrated that after knockdown of SIPA1 in HT115 and Caco-2 colorectal cancer cell lines, the potential of cancer cells to invade, adhere and migrate was increased compared to the control group, while the ability to proliferate was decreased (34). This suggests that SIPA1 may have an active role during progression of colorectal adenocarcinoma.

Cervical cancer. Two SNPs in SIPA1 (rs931127, 313G >A and rs $746429,2760 \mathrm{G}>\mathrm{A}$ ) are potentially related to an increased risk of nodal metastasis in cervical cancer. The $\mathrm{G}$ allele at both rs931127 and rs746429 in SIPA1 was associated with nodal disease in cases and controls. In terms of tumour size, patients with smaller stage IB1 tumours having the G allele showed an increased risk of nodal metastases at SIPA1 rs746429 and at rs931127, but the correlated trend between polymorphisms in SIPA1 and nodal metastasis were not significant in Stage IB2 tumours (which are larger lesions). The G allele in SIPA1 at both rs746429 and rs931127 was significantly related to nodal disease in patients without lymphovascular invasion (LVI), which was considered as an independent poor prognostic factor in cervical cancer patients. The GG genotype was associated with a markedly higher risk of nodal disease in both SNPs of SIPA1 in patients without LVI. Histologically, SNPs in SIPA1 rs746429 and rs931127 were not related to histology types (adenocarcinoma or squamous cancer). Moreover, SNPs of SIPA1 made no difference in the overall survival of cervical cancer patients (20).

Lung cancer. Previous studies have focused on the polymorphism of SIPA1 in lung cancer development and metastasis. Three SNPs were found to be involved in lung cancer, rs931127 A>G, rs2448490 G>A and rs3741379 G>T $(24,25)$. In a southern Chinese population, a high frequency of the $\mathrm{G}$ allele at rs931127 was significantly correlated with the risk of lung cancer, but no significant connection was observed for the other two SNPs. From the perspective of tumour staging and grading, the fusion of the $\mathrm{G}$ allele in rs 931127 was also associated with worse clinical stage, nodal metastasis and distal metastasis. The SNP rs931127 A>G in the promoter of SIPA1 was highly associated with tumourogenesis and metastasis of lung cancer (25). Another study suggested that $G$ allele fusion in SNP rs931127 A>G was significantly correlated with more serious progression free survival (PFS) in the patients with non-small cell lung cancer (NSCLC) (24). Therefore the SIPA1 SNP rs931127 A>G may be identified as an independent prognostic predictive factor for progression free survival (PFS) in NSCLC patients $(24,25)$. 
Gastric cancer. There has been little previous research regarding the role of SIPA1 in gastric cancer. A single study demonstrated both mRNA level (detect by qPCR) and protein expression (detected by western blotting) of SIPA1 in gastric tumour tissues was lower than in adjacent normal tissues. However, on the contrary, IHC that positive staining of SIPA1 was significantly higher in gastric cancer tissue than in adjacent normal tissue (35). Positive staining of SIPA1 in the tumour from gastric cancer patients was markedly associated with the degree of differentiation, lymph node metastases and clinical grading (35). The expression and function of SIPA1 in gastric cancer and its mechanisms of action need to be further explored.

Melanoma. Limited research regarding the role of SIPA1 in melanoma has been conducted. One study indicated that in fast-growing melanoma there was significant overexpression of SIPA1, resulting in the inactivation of Rap1 and aggressive melanoma cell models. When knockdown down of SIPA1 in these melanoma models was carried out, adhesion capability was enhanced but clonogenic potential and migration ability were reduced (36). These data suggest that SIPA1 interacts with Rapl and may have a complex role in the regulation of melanoma development and metastasis.

\section{Conclusion}

Although it has been nearly 30 years since the discovery of SIPA1, there has been a scarcity of research as regards the role of SIPA1 in cancer development or prognosis. In almost all types of cancers studied, SIPA1 was associated with lymph node metastasis, which suggests that SIPA1 might be a marker of, or at least associated with, cancer development and hence patients' prognosis. Whether it could prove to be a reliable biomarker for prognosis remains to be seen and further work is necessary.

Numerous studies have demonstrated that interactions between SIPA1 and several other molecules, known to be involved in control of cell growth and division, could offer a potential model for studying the process of cancer metastasis. In relation to this, the role of SIPA1 in Rap1 signalling pathway may also shed light on the regulation of this pathway in metastatic disease and provide a novel target in the future.

We can conclude that SIPA1 may be a new target for cancer therapy in those patients with tumours which have resistance to current chemotherapy agents. But so far there were no ongoing or potential clinical trials for targeting SIPA1. Potential inhibitors to SIPA1 could have a direct bearing on its putative role as a therapeutic target, the importance of which has yet to be determined.

\section{Acknowledgements}

Not applicable.

\section{Funding}

No funding was received.

\section{Availability of data and materials}

Not applicable.

\section{Authors' contributions}

CL, TAM, RH and WGJ made substantial contributions to conception and design of the review. CL, TAM, RH and WGJ were involved in drafting, revising and intellectual content. All authors were given final approval of the version to be published.

\section{Ethics approval and consent to participate}

Not applicable.

\section{Patient consent for publication}

Not applicable.

\section{Competing interests}

The authors declare that they have no competing interests.

\section{References}

1. Paget S: The distribution of secondary growths in cancer of the breast. Lancet 133: 571-573, 1889.

2. Fidler IJ: The pathogenesis of cancer metastasis: The 'seed and soil' hypothesis revisited. Nat Rev Cancer 3: 453-458, 2003.

3. Hanahan D and Weinberg RA: Hallmarks of cancer: The next generation. Cell 144: 646-674. 2011.

4. Valastyan S and Weinberg RA: Tumor metastasis: Molecular insights and evolving paradigms. Cell 147: 275-292, 2011.

5. Lee EY and Muller WJ: Oncogenes and tumor suppressor genes. Cold Spring Harb Perspect Biol 2: a003236, 2010.

6. Seoane $J$ and Gomis RR: TGF- $\beta$ family signaling in tumor suppression and cancer progression. Cold Spring Harb Perspect Biol 9: a022277, 2017.

7. Dhillon AS, Hagan S, Rath O and Kolch W: MAP kinase signalling pathways in cancer. Oncogene 26: 3279-3290, 2007.

8. Logan CY and Nusse R: The Wnt signaling pathway in development and disease. Annu Rev Cell Dev Biol 20:781-810, 2004.

9. Ranganathan P, Weaver KL and Capobianco AJ: Notch signalling in solid tumours: A little bit of everything but not all the time. Nat Rev Cancer 11: 338-351, 2011.

10. Sulzmaier FJ, Jean C and Schlaepfer DD: FAK in cancer: Mechanistic findings and clinical applications. Nat Rev Cancer 14: 598-610, 2014.

11. Vanhaesebroeck B, Guillermet-Guibert J, Graupera M and Bilanges B: The emerging mechanisms of isoform-specific PI3K signalling. Nat Rev Mol Cell Biol 11: 329-341, 2010.

12. Goncalves MD, Hopkins BD and Cantley LC: Phosphatidylinositol 3-kinase, growth disorders, and cancer. N Engl J Med 379: 2052-2062, 2018.

13. Hattori M: SIPA1 (signal-induced proliferation-associated 1). Atlas of Genetics and Cytogenetics in Oncology and Haematology, 2011.

14. Hattori M, Tsukamoto N, Nur-e-Kamal MS, Rubinfeld B, Iwai K, Kubota $\mathrm{H}$, Maruta $\mathrm{H}$ and Minato N: Molecular cloning of a novel mitogen-inducible nuclear protein with a Ran GTPase-activating domain that affects cell cycle progression. Mol Cell Biol 15: $552-560,1995$

15. Kurachi H, Wada Y,TsukamotoN, Maeda M,Kubota H,Hattori M, Iwai K and Minato N: Human SPA-1 gene product selectively expressed in lymphoid tissues is a specific GTPase-activating protein for Rap1 and Rap2. Segregate expression profiles from a rap1GAP gene product. J Biol Chem 272: 28081-28088, 1997.

16. Roberts MR, Hong CC, Edge SB, Yao S, Bshara W, Higgins MJ, Freudenheim JL and Ambrosone CB: Case-only analyses of the associations between polymorphisms in the metastasis-modifying genes BRMS1 and SIPA1 and breast tumor characteristics, lymph node metastasis, and survival. Breast Cancer Res Treat 139: 873-885, 2013.

17. Crawford NP, Ziogas A, Peel DJ, Hess J, Anton-Culver H and Hunter KW: Germline polymorphisms in SIPA1 are associated with metastasis and other indicators of poor prognosis in breast cancer. Breast Cancer Res 8: R16, 2006. 
18. Gaudet MM, Hunter K, Pharoah P, Dunning AM, Driver K, Lissowska J, Sherman M, Peplonska B, Brinton LA, Chanock S and Garcia-Closas M: Genetic variation in SIPA1 in relation to breast cancer risk and survival after breast cancer diagnosis. Int J Cancer 124: 1716-1720, 2009.

19. Hsieh SM, Look MP, Sieuwerts AM, Foekens JA and Hunter KW: Distinct inherited metastasis susceptibility exists for different breast cancer subtypes: A prognosis study. Breast Cancer Res 11: R75, 2009.

20. Brooks R, Kizer N, Nguyen L, Jaishuen A, Wanat K, Nugent E, Grigsby P, Allsworth JE and Rader JS: Polymorphisms in MMP9 and SIPA1 are associated with increased risk of nodal metastases in early-stage cervical cancer. Gynecol Oncol 116: 539-543, 2010.

21. Pei R, Xu Y, Wei Y, Ouyang T, Li J, Wang T, Fan Z, Fan T, Lin B and Xie Y: Association of SIPA1 $545 \mathrm{C}>\mathrm{T}$ polymorphism with survival in Chinese women with metastatic breast cancer. Front Med 7: 138-142, 2013.

22. Ugenskienė R, Myrzaliyeva D, Jankauskaitė R, Gedminaitė J, Jančiauskienė R, Sepetauskienè E and Juozaitytė E: The contribution of SIPA1 and RRP1B germline polymorphisms to breast cancer phenotype, lymph node status and survival in a group of Lithuanian young breast cancer patients. Biomarkers 21: 363-370, 2016

23. Hsieh SM, Smith RA, Lintell NA, Hunter KW and Griffiths LR Polymorphisms of the SIPA1 gene and sporadic breast cancer susceptibility. BMC Cancer 9: 331, 2009.

24. Gdowicz-Kłosok A, Giglok M, Drosik A, Suwiński R and Butkiewicz D: The SIPA1 $-313 \mathrm{~A}>\mathrm{G}$ polymorphism is associated with prognosis in inoperable non-small cell lung cancer. Tumour Biol 36: 1273-1278, 2015.

25. Xie C, Yang L, Yang X, Yang R, Li Y, Qiu F, Chen M, Fang W, Bin X, Deng J, et al: Sipal promoter polymorphism predicts risk and metastasis of lung cancer in Chinese. Mol Carcinog 52 (Suppl 1): E110-E117, 2013.

26. Zhang Y, Gong Y, Hu D, Zhu P, Wang N, Zhang Q, Wang M, Aldeewan A, Xia H, Qu X, et al: Nuclear SIPA1 activates integrin betal promoter and promotes invasion of breast cancer cells. Oncogene 34: 1451-1462, 2015.

27. Su L, Hattori M, Moriyama M, Murata N, Harazaki M, Kaibuchi K and Minato N: AF-6 controls integrin-mediated cell adhesion by regulating Rapl activation through the specific recruitment of Rap1GTP and SPA-1. J Biol Chem 278: 15232-15238, 2003.
28. Noda Y, Horikawa S, Furukawa T, Hirai K, Katayama Y, Asai T, Kuwahara M, Katagiri K, Kinashi T, Hattori M, et al: Aquaporin-2 trafficking is regulated by PDZ-domain containing protein SPA-1. FEBS Lett 568: 139-145, 2004.

29. Alsarraj J, Faraji F, Geiger TR, Mattaini KR, Williams M, Wu J, Ha NH, Merlino T, Walker RC, Bosley AD, et al: BRD4 short isoform interacts with RRP1B, SIPA1 and components of the LINC complex at the inner face of the nuclear membrane. PLoS One 8: e80746, 2013.

30. Farina A, Hattori M, Qin J, Nakatani Y, Minato N and Ozato K: Bromodomain protein Brd4 binds to GTPase-activating SPA-1, modulating its activity and subcellular localization. Mol Cell Biol 24: 9059-9069, 2004.

31. Park YG, Zhao X, Lesueur F, Lowy DR, Lancaster M, Pharoah P, Qian X and Hunter KW: Sipa1 is a candidate for underlying the metastasis efficiency modifier locus Mtes1. Nat Genet 37: 1055-1062, 2005

32. Shimizu Y, Hamazaki Y, Hattori M, Doi K, Terada N, Kobayashi T, Toda Y, Yamasaki T, Inoue T, Kajita Y, et al: SPA-1 controls the invasion and metastasis of human prostate cancer. Cancer Sci 102: 828-836, 2011.

33. Takahara T, Kasamatsu A, Yamatoji M, Iyoda M, Kasama H, Saito T, Takeuchi S, Endo-Sakamoto Y, Shiiba M, Tanzawa H and Uzawa K: SIPA1 promotes invasion and migration in human oral squamous cell carcinoma by ITGB1 and MMP7. Exp Cell Res 352: 357-363, 2017.

34. Ji K, Ye L, Toms AM, Hargest R, Martin TA, Ruge F, Ji J and Jiang WG: Expression of signal-induced proliferation-associated gene 1 (SIPA1), a RapGTPase-activating protein, is increased in colorectal cancer and has diverse effects on functions of colorectal cancer cells. Cancer Genomics Proteomics 9: 321-327, 2012.

35. Li JY, Wang JB, Liu CB, Ma DL and Ma JH: Dynamic relationship between SIPA1 gene and protein expression and the development of gastric cancer. Genet Mol Res 16, 2017.

36. Mathieu V, Pirker C, Schmidt WM, Spiegl-Kreinecker S, Lötsch D, Heffeter P, Hegedus B, Grusch M, Kiss R and Berger W: Aggressiveness of human melanoma xenograft models is promoted by aneuploidy-driven gene expression deregulation. Oncotarget 3: 399-413, 2012. 